\title{
CONCRETE STRENGTH EVALUATION TECHNIQUE USING PIEZOELECTRIC GUIDED-WAVE PROPAGATIONS
}

\author{
$\underline{\text { Dong Jin Kim }}{ }^{1}$, Changgil Lee ${ }^{2}$, Seok Inn Hong ${ }^{3}$, and Seunghee Park ${ }^{2 *}$ \\ ${ }^{1}$ Department of u-City Design \& Engineering, Sungkyunkwan University, Suwon, Korea \\ ${ }^{2}$ Department of Civil \& Environmental Engineering, Sungkyunkwan University, Suwon, Korea \\ ${ }^{3}$ Department of Disaster prevention Team, Daewoo E\&C, Suwon, Korea \\ * Corresponding author (shparkpc@skku.edu)
}

\begin{abstract}
Recently, novel methods using piezoelectric materials to monitor the strength development of concrete during curing process have been reported. However, these methods showed some limitations to obtain an absolute concrete strength in real-world. In order to estimate the absolute strength of the concrete, the concrete strength monitoring method using guided- wave propagation is proposed in this study. Because the wave signals are varied by the change of mechanical properties of structure, the strength of the concrete can be estimated. Moreover, to obtain the absolute strength of the concrete, specific strength equations are derived using the features extracted from the wave signal variations according to the strength development of the concrete. To verify the effectiveness of the proposed approach, a series of experimental studies using miscellaneous concrete specimens have been carried out. The guided-wave signal was measured by embedded piezoelectric sensors using self-sensing method. The maximum propagation power was extracted from the each signal, and the strength estimation equation was derived from the relationship between maximum propagation power and measured compressive strength. Finally, further research issues will be discussed for real-world implementation of the proposed approach.
\end{abstract}

Keywords: Concrete Strength Estimation, Guided-Wave Propagation, Embedded Piezoelectric Sensor, Linear Regression

\section{INTRODUCTION}

Recently, there has been increasing demand for super highrise buildings or super long span bridges. That means there is a big need for large amount of concrete structures in our civil environments. However, the concrete structures might be susceptible to brittle fracture if their curing process is inadequate. Therefore, to prevent this problem, it is very essential to predict the absolute strength of the concrete material nondestructively. As one of solution, this study proposes a new concept of real-time strength monitoring technique based on piezoelectric materials. In this study, efforts to confirm the applicability of the guided-wave using embedded piezoelectric sensor have focused monitoring on the strength of concrete structures.

\section{GUIDED-WAVE BASED STRENGTH ESTIMATION MODEL}

The guided-wave signal represents the mechanical properties of host structure. The mechanical properties change is caused by the variation of strength dominantly in curing process. Therefore the guided-wave signals vary according to strength variation of host concrete structure. Recently, Lee et al proposed a self-sensing based measurement system [1] and the Song et al developed the smart aggregate to embed piezoelectric sensor into the concrete [2]. The guided-wave signals which mean the response about tone-burst input signals in this study can be measured with single embedded piezoelectric sensor by using this method.

The test was performed to the unreinforced concrete structure and sensor was embedded as shown in Fig. 1. 


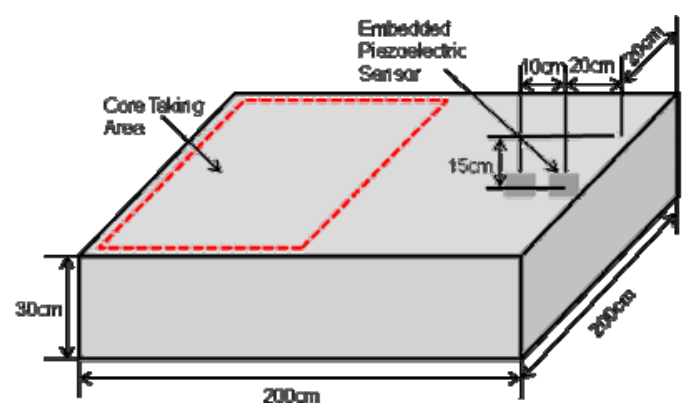

Fig. 1 Diagram of test specimen

The test was processed at 2, 3, 5, 7, 10, 14, and 28 day after casting and the results of each day is shown in Fig. 2. The maximum power of guided wave signal is getting higher according to the strength development.

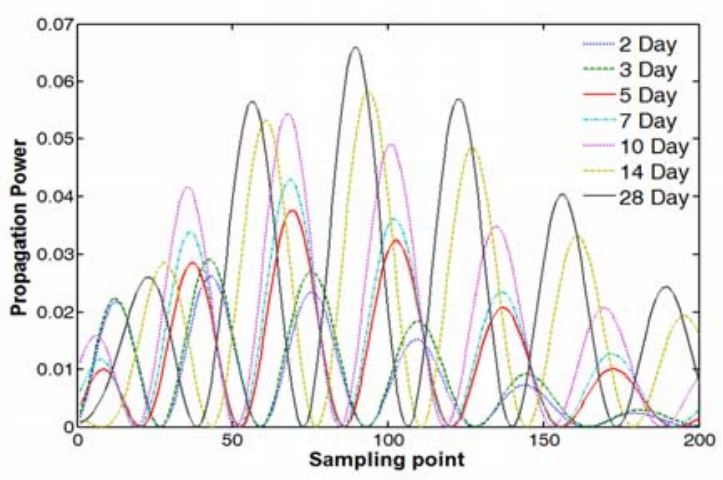

Fig. 2 Propagation power at each curing age

Also the compressive strength of test specimen was measured by core test. The value of maximum propagation power was extracted and matched with measured compressive strength using linear regression method to derive strength estimation model as shown in Eq.(1).

$$
\begin{aligned}
& S(M P a)=333.2 P_{w}+8.692 \\
& R^{2}=0.9623
\end{aligned}
$$

Where the $\mathrm{S}$ represents estimation strength and the $\mathrm{P}_{\mathrm{w}}$ represents the maximum propagation value.

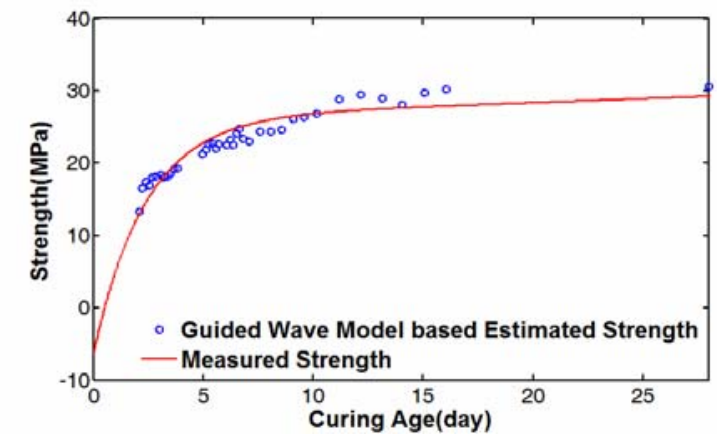

Fig. 3 Propagation power at each curing age
The estimated strength using this guided-wave based strength estimation model is shown in Fig 3 . The line is the measured strength curve based on core testing and the dot means the estimated strength at each curing age. So it is confirmed the strength estimation model can estimate the strength of the concrete structure in this case.

\section{CONCLUSION}

This study proposed the real-time concrete strength monitoring technique using the maximum propagation power of guided-wave. To estimate continuously the absolute strength of concrete during its curing process, the guided-wave methods which are normally used to detect structural damage nondestructively, was applied. The extracted strength features were the maximum propagation power variation of the guided-wave signals. The strength estimation model was derived by matching the maximum propagation power with measured compressive strength using linear regression method. Conclusively, it is experimentally confirmed that the guided-wave based strength estimation model can be the one of solution to obtain the absolute strength of the concrete structures.

\section{ACKNOWLEDGEMENT}

This study was supported by the u-City Master and Doctor Support Project funded by Ministry of Land, Transport and Maritime Affairs (MLTM), Daewoo E\&C, and the Nuclear Research \& Development of the Korea Institute of Energy Technology Evaluation and Planning (KETEP) grant funded by the Korea government Ministry of Knowledge Economy in Korea(20101610004J).

\section{REFERENCES}

[1] Lee, S.J., and Sohn, H., "Active self-sensing scheme development for structural health monitoring", Smart Materials and Structures, Vol. 15(6), pp. 1734-1746 , 2006. [2] Song, G., Gu, H., and Mo, Y.L., "Smart aggregates: Multi-functional sensors for concrete structures - A tutorial and a review", Smart materials and Structures, Vol. 17(3), 033001, 2008. 